\title{
A Correlative Study of HRTEM, HAADF-STEM, and STEM-EELS Spectrum Imaging for Biphasic Electrochemically Active $\mathrm{TiO}_{2}$
}

\author{
Chang Wan Han ${ }^{1}$, Vinodkumar Etacheri ${ }^{2}$, Chulgi Nathan Hong ${ }^{2}$, Vilas G. Pol ${ }^{2}$, and Volkan Ortalan ${ }^{1}$ \\ 1. School of Materials Engineering, Purdue University, West Lafayette, IN, USA \\ 2. School of Chemical Engineering, Purdue University, West Lafayette, IN, USA
}

Nanocrystalline $\mathrm{TiO}_{2}$ polymorphs have been widely studied as candidate materials for the energy harvesting and storage applications, including photovoltaic devices and rechargeable batteries due to its excellent performances as well as low cost [1-3]. Interestingly, it has been reported that a mixture of the polymorphs shows improved photocatalytic activity compared to that of a single $\mathrm{TiO}_{2}$ polymorph $[4,5]$. D. C. Hurum et al. found the improved activity of the biphasic $\mathrm{TiO}_{2}$, Degussa P25 - a mixture of anatase and rutile, is due to the synergetic charge transfer effect between them [6]. Recently, we also found that the synergetic effect in a biphasic $\mathrm{TiO}_{2}$ anode significantly improves the capacity and the rate performance of lithium ion batteries. Considering the fact that the biphasic form of $\mathrm{TiO}_{2}$ outperforms single polymorphic phases in the energy-related applications, various forms of biphasic materials would be further developed. Since the polymorphs in the biphasic materials have identical composition with each other, distribution of the constituent polymorph in a biphasic material is not readily obtainable at high spatial resolution. Therefore, most of reports just provide relative amount of constituent phases determined by powder X-ray diffraction and/or Raman spectroscopy and high-resolution transmission electron microscopy (HRTEM) images with low magnification in which the spatial distribution of the polymorphs are not clearly shown [5,7,8]. In this respect, a systematic approach to characterize the biphasic materials is essential.

In this study, we performed a correlative approach, including HRTEM, high-angle annular dark field (HAADF) scanning TEM (STEM), and STEM electron energy loss spectroscopy (EELS) spectrum imaging to characterize a biphasic (bronze + anatase) $\mathrm{TiO}_{2}$ nanosheets showing excellent electrochemical performance in lithium ion batteries. HRTEM images collected at a high magnification (over $800 \mathrm{kX}$ ) were used to characterize atomic structures of the polymorphs [Fig. 1a]. Although HRTEM has been a good approach for investigating atomic structures, it may not be suitable for morphological studies due to the complicated contrast mechanism. Moreover, a limited field of view in HRTEM at high magnification makes difficult to get the distribution of the comprising phases. Therefore, we performed HAADF-STEM imaging, providing morphological features of nanocrystalline $\mathrm{TiO}_{2}$ polymorphs [Fig. 1b]. Finally, EELS spectrum imaging was performed to reveal the relative distribution of each $\mathrm{TiO}_{2}$ polymorph in the biphasic $\mathrm{TiO}_{2}$ nanosheets. Unique high-resolution electron energy loss near edge structures of $\mathrm{TiO}_{2}$ polymorphs, collected by the JEOL ARM200CF, an aberration corrected STEM with a cold field emission gun allowed us to determine how nanocrystallites of each polymorph are distributed. Collected spectrum images were de-noised by principal component analysis (PCA) and the distribution was obtained by multiple linear least square (MLLS) fitting [Fig. 1c]. The results of a correlative analysis will be provided and the correlation between the structural features and the exceptional performance of the biphasic $\mathrm{TiO}_{2}$ nanosheets will be discussed. 
References

[1] B. O'Regan et al, Nature. 353 (1991) p. 737-740.

[2] A.G. Dylla et al, Acc. Chem. Res. 46 (2013) p. 1104-1112.

[3] V. Etacheri et al, ACS Nano. 8 (2014) p. 1491-1499.

[4] A.G. Agrios et al, Langmuir. 19 (2003) p. 1402-1409.

[5] Y. Chimupala et al, RCS Adv. 4 (2014) p. 48507-48515.

[6] D.C. Hurum et al, J. Phys. Chem. B. 107 (2003) p. 4545-4549.

[7] G. Li et al, Dalt. Trans. (2009) p. 10078-10085.

[8] M. Estruga et al, Nanotechnology. 20 (2009) p. 125604.

[9] We thank the Purdue University and School of Chemical Engineering for their generous start-up funding.

(a) HRTEM

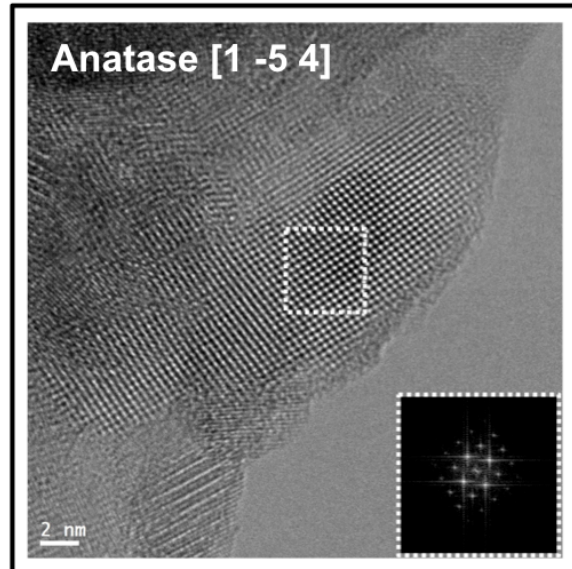

(b) HAADF-STEM
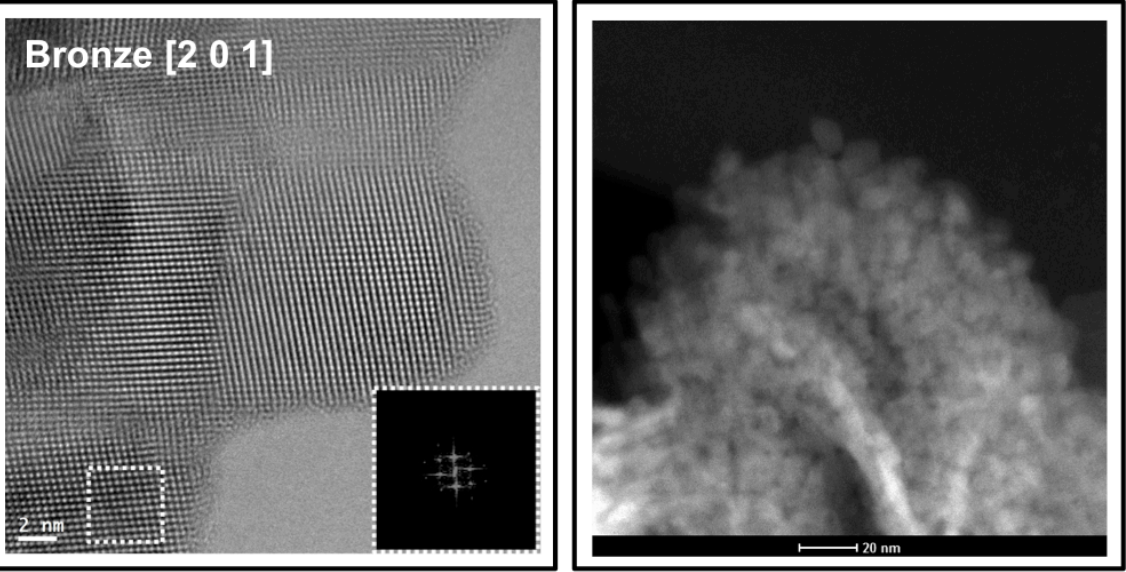

(c) STEM-EELS Spectrum Imaging

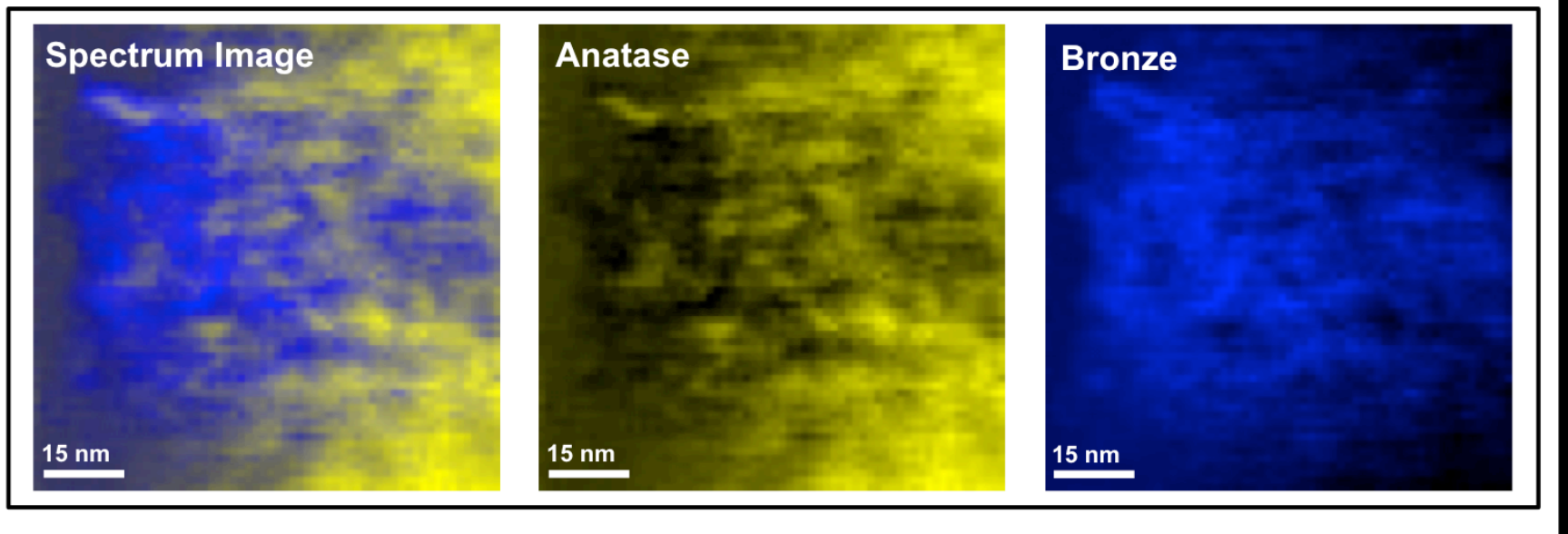

Figure 1. Results of correlative electron microscopy studies for the biphasic $\mathrm{TiO}_{2}$ : HRTEM images showing the atomic structures of $\mathrm{TiO}_{2}$ nanocrystallites (Fig. 1a), HAADF-STEM image for morphologies (Fig. 1b), and STEM-EELS spectrum images revealing the distribution of the constituent phase (Fig. 1c) 